\title{
Primary health eye care: evaluation of the competence of medical students in performing fundoscopy with the direct ophthalmoscope
}

\author{
${ }^{a}$ Van Velden JS, MBChB (Stell), DipOphth (SA), FCOphth (SA), MMED Ophth (UCT) a Cook C, MBChB (UCT), FCOphth (SA), FRCOphth, MPH (UCT)

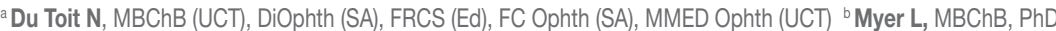 \\ a Division of Ophthalmology, Groote Schuur Hospital, University of Cape Town, Cape Town, South Africa \\ ${ }^{\mathrm{b}}$ School of Public and Family Medicine, University of Cape Town, Cape Town, South Africa \\ Correspondence to: Dr JS van Velden, e-mail: hvanvelden@yahoo.com \\ Keywords: primary health eye care; teaching fundoscopy; essential basic medical skill; medical student training
}

Abstract

SA Fam Pract 2010;52(4):341-343

Background: To evaluate the skill of fifth-year medical students at the University of Cape Town in the performance of fundoscopy at the end of their ophthalmology rotation.

Methods: The design was a prospective cohort study. The study was conducted at Groote Schuur Hospital in Cape Town. The ability of fifth-year medical students to perform fundoscopy on mannequin heads fitted with fundus photographs using direct ophthalmoscopes was evaluated. The outcome measures used were ability to see the fundus and ability to recognise fundus pathology.

Results: The fundus photographs could be seen in $95 \%$ of cases. The correct diagnosis could be made in only $57 \%$ of cases.

Conclusion: Medical students at the University of Cape Town need more than the current one week of practical ophthalmology training to improve their fundoscopy skills.

(P) Peer reviewed. (Submitted: 2009-10-17, Accepted: 2010-01-30). ๑ SAAFP

\section{Background}

Fundoscopy is considered an essential basic clinical skill for family physicians. Glaucoma and diabetic retinopathy are leading causes of blindness globally and in Southern Africa. Globally, 4.5 million people are blinded by glaucoma, and 1.8 million by diabetic retinopathy. ${ }^{1}$ Visual loss can be prevented in both glaucoma and diabetic retinopathy if the diseases are diagnosed and treated early. An adequate examination of the fundus is necessary to recognise both these conditions in the primary care setting. Examination of the optic disc with a direct ophthalmoscope using a cut point of 0.7 for the vertical cup:disc ratio is recommended for the detection of glaucoma. ${ }^{2}$ Similarly, diabetic retinopathy can only be detected on fundoscopy, and the visual impairment due to diabetic retinopathy is preventable if the retinopathy can be identified by screening and if treatment is implemented at an early stage in the disease..$^{3-5}$ Such screening and early treatment can reduce the risk of blindness by up to $56 \%{ }^{6}$

In addition, sight-threatening and life-threatening conditions such as cytomegaloviral retinitis occurring in patients with AIDS and papilloedema occurring in patients with raised intracranial pressure, which need to be recognised by family physicians, can only be seen on fundoscopy.
Whilst alternative instruments and techniques are available for fundal examination by ophthalmologists (panoptic ophthalmoscope, slit lamp biomicroscopy, and indirect ophthalmoscope), fundoscopy with a direct ophthalmoscope remains the preferred choice for primary care clinicians. Mastering the skill of fundoscopy requires practice, and it may be one of the most difficult skills required for the examination of patients. ${ }^{7-9}$

There have been a number of studies evaluating fundoscopy skills amongst medical students and primary care physicians. Cordeiro et al have shown the benefit of formal training of medical students by comparing the skills in medical students randomised either to receive or not to receive formal instruction in fundoscopy. ${ }^{7}$ Lippa et al found that, after an initially acceptable level of performance in fundoscopy by medical students who received training in fundoscopy in their second year of study, there was a deterioration in their skills in the following year. ${ }^{8}$ After a reinforcement course in their fourth year of study, however, there was a marked improvement in their skills. ${ }^{8}$ In another study, Gupta et al found that the confidence of medical students to perform fundoscopy adequately was low after their brief exposure to formal instruction during their medical training. ${ }^{9}$ This consisted of one week of ophthalmology lectures and three hours of practical instruction in their first year, a four-hour clinical skills session in their second 
year, and a one-week rotation in ophthalmology in their third year, which included some lectures and a 2.5-day allocation in the ophthalmology clinic. The authors found that the students expressed a need for more practice and training in fundoscopy. ${ }^{9}$ Two studies looked at the ability of general practitioners to screen for diabetic retinopathy with a direct ophthalmoscope. In the first study, Khandekar et al found that general practitioners who had no further training after their medical school training were unable to screen for diabetic retinopathy and needed further training before they could do so. ${ }^{10}$ In the second study, by Verma et al., the practitioners had a further short period of training and were then found to have fundoscopy skills of an acceptable standard to allow their participation in a diabetic screening programme. ${ }^{11}$

From these studies it would seem that the three factors that are important for providing adequate training in fundoscopy are:

1. Formal instruction in the technique.

2. Adequate time allocated to practise the skill.

3. Opportunity for repeat refresher training.

Students at the University of Cape Town receive formal instruction in the technique of fundoscopy at the beginning of their ophthalmology rotation in their fifth year of study. However, only one week is allocated for them to practise the skill in the eye clinic, and they do not have opportunity for refresher training.

This study was undertaken to evaluate the fundoscopy skills of students at the University of Cape Town who have completed their fifth-year ophthalmology rotation.

\section{Method}

Ethical approval was obtained from the University of Cape Town Health Science Faculty's Research Ethics Committee.

Fifth-year students doing their ophthalmology rotation in 2007 and 2008 participated in the study. Participation in the study was voluntary. At the beginning of their rotation, the students received formal instruction in the technique of fundoscopy using a direct ophthalmoscope. The two types of direct ophthalmoscopes that are recommended for use by medical students during their rotation are a conventional direct ophthalmoscope with a focusing lens, and a smaller penlight ophthalmoscope with no focusing lens. They were encouraged to practise the skill with both ophthalmoscopes when examining patients in the clinic. At the end of their rotation, they were evaluated using mannequin heads fitted with fundus slides. The mannequin heads are designed for training and practice in fundoscopy. The prosthetic eyes of the mannequin heads have an artificial cornea, a pupil with a diameter of $6 \mathrm{~mm}$, and a slide of a photographic image of the human fundus on a scale of 1:1. The slides are placed at the appropriate distance from the cornea to simulate emmetropia (Figure 1). Students with a refractive error were requested to use their ametropic correction, i.e. glasses or contact lenses, during the performance of fundoscopy.

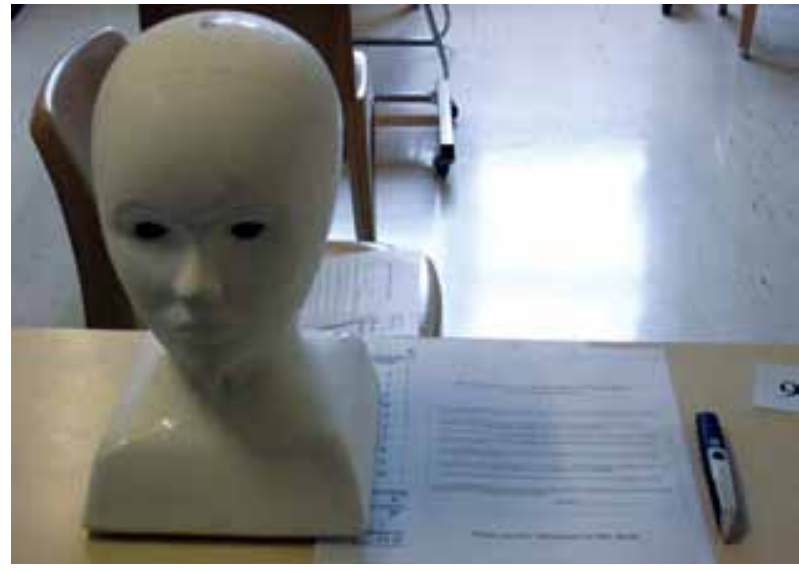

Ten slides of five fundal conditions were used, with two similar but non-identical slides of each condition:

1. Normal fundus (disc and macula)

2. Descending optic atrophy

3. Cupped glaucomatous disc

4. Swollen disc

5. Diabetic maculopathy

The ten mannequin heads were placed on separate tables in random order with one of the two types of ophthalmoscopes, ensuring that each diagnosis, represented by a pair of similar slides, was accompanied by both types of ophthalmoscopes. Two minutes were allocated for each station. The students were required to answer two questions at each station:

- Can you see the fundus (yes/no)

- What is the diagnosis?

Data was captured on a custom-designed template. Descriptive statistics were used to reflect the results of this study, namely the number and percentage of fundal slides visualised, and the number and percentage of correct diagnoses made.

\section{Results}

\section{Subjects recruited}

A total of 173 fifth-year medical students were recruited into the study in 2006 and 2007. All of the students agreed to participate in the study.

\section{Ability to see the fundus photographs}

Of a total of 1730 fundus photographs viewed, the students were able to visualise the fundi of 1647 (95.2\%) of the photographs with a direct ophthalmoscope. The students performed equally well with both types of ophthalmoscopes $(p=0.7)$.

\section{Overall ability to make the correct diagnosis}

The correct diagnosis was made in 991 (57.3\%) of cases.

\section{Ability to make specific diagnoses}

Table I shows the ability of the students to correctly diagnose specific conditions. 
Table I: Ability of the students to diagnose specific conditions

\begin{tabular}{|l|c|}
\hline Diagnosis & $\begin{array}{c}\text { Correct diagnosis } \\
\text { Number (\%) }\end{array}$ \\
\hline Normal fundus & $154(44.5)$ \\
\hline Descending optic atrophy & $196(56.6)$ \\
\hline Swollen disc & $191(55.2)$ \\
\hline Cupped disc & $208(60.1)$ \\
\hline Diabetic maculopathy & $284(82)$ \\
\hline
\end{tabular}

\section{Discussion}

\section{Ability to see the fundus photographs}

The students said they could see the fundus photographs in $95 \%$ of cases. This compares favourably with a study done by Lippa et al., who found that 72 to $82 \%$ of the students in their study could visualise various parts of the fundus. ${ }^{8}$ This would suggest that the students managed to master the technique of using the ophthalmoscope to obtain a view of the fundus.

\section{Ability to make a diagnosis}

Overall, the students were able to make the correct diagnosis in only $57 \%$ of cases. They were able to recognise diabetic retinopathy in $82 \%$ of cases, but were only able to recognise glaucoma cupping in $60 \%$ of cases. Glaucoma and diabetic retinopathy are leading causes of blindness, both globally and locally, ${ }^{1}$ and early recognition on fundoscopy is important if visual loss is to be prevented. Also, missing optic atrophy or papilloedema as manifestations of systemic diseases, such as an intracranial tumour, can have serious consequences.

\section{Shortcomings of this study}

We used mannequin heads with prosthetic eyes for this study. We would have preferred to have tested the students' abilities to do fundoscopy and to recognise fundal pathology in real patients. This was unfortunately neither practical nor feasible.

We assessed the skills of the medical students in fundoscopy just once, immediately after their ophthalmology rotation. Lippa et al evaluated the retention of fundoscopy skills in medical students and found an erosion of skills over their three-year evaluation period. ${ }^{8}$ They also found an improvement in fundoscopy in these students after a brief refresher course. It would be of interest to re-evaluate the students again after a one to two-year period, and to evaluate the impact of refresher training.

\section{Conclusion}

Fundoscopy is an essential basic clinical skill for a family physician. Three factors that are important for optimising the opportunity for students to attain good competence in this skill are formal training in the skill, adequate opportunity to practise the skill, and repeat refresher training later in the course. Medical students at the University of Cape Town currently receive formal training in fundoscopy at the beginning of their ophthalmology rotation in their fifth year of study, but subsequently only have one week in which to practise the skill and no opportunity for further refresher training. Whilst they say that they are able to see the fundus photograph of prosthetic eyes in mannequin heads designed for training and practice in fundoscopy in $95 \%$ of cases, they could make the correct diagnosis in only $57 \%$ of cases. It would seem, therefore, that the present MBChB curriculum at the University of Cape Town provides inadequate opportunity for students to practise and master the skill of fundoscopy. It is recommended that the curriculum be revised to allow for:

1. Formal training in fundoscopy earlier in the course. This could be given during the clinical skills block in the third year.

2. Increased time allocated to practise the skill. This could be provided during the medicine block in the fourth year and during an expanded two-week ophthalmology block in the fifth year.

3. Refresher retraining. This could be given again at the beginning of the two-week ophthalmology block in the fifth year.

\section{Conflict of interest}

We declare that we have no financial or personal relationships that may have inappropriately influenced us in writing this paper.

\section{References}

1. World Health Organization. State of the world's sight. Vision 2020 - The right to sight. Geneva: WHO; 2005.

2. Cook C, Cockburn N, Van der Merwe J, Ehrlich R. Cataract and glaucoma case detection for Vision 2020 programmes in Africa - an evaluation of six possible screening tests. J Glaucoma. In press.

3. Bailey CC, Sparrow JM, Grey RHB, et al. National diabetic retinopathy laser treatment audit. III. Clinical outcomes. Eye 1999;13:151-9.

4. Harvey JN, Craney L, Nagendran S, Ng CS. Towards comprehensive populationbased screening for diabetic retinopathy: operation of the North Wales diabetic retinopathy screening programme using a central patient register and various screening methods. J Med Screen 2006;13:87-92.

5. Read O, Cook C. Retinopathy in diabetic patients evaluated at a primary care clinic in Cape Town. S Afr Med J 2007;97:941-3.

6. Rohan TE, Frost CD, Wald NJ. Prevention of blindness by screening for diabetic retinopathy: a quantitive assessment. BMJ 1989;299:1198-201.

7. Cordeiro MF, Jolly BC, Dacre JE. The effect of formal instruction in ophthalmoscopy on medical student performance. Med Teach 1993;15:321-5.

8. Lippa LM, Boker J, Duke A, Amin A. A novel 3-year pilot study of medical students' acquisition and retention of screening eye examination skills. Ophthalmology 2006;113:984.

9. Gupta RR, Lam W. Medical students' self-confidence in performing direct ophthalmoscopy in clinical training. Can J Ophthalmol 2006;41:169-74.

10. Khandekar R, Shah S, Lawatti JA. Retinal examination of diabetic patients: knowledge, attitudes and practices of physicians in Oman. Eastern Mediterranean Health Journal 2008;14.

11 Verma L, Prakash G, Tewari HK, et al. Screening for diabetic retinopathy by non-ophthalmologists: an effective public health tool. Acta Ophthalmol Scand 2003;81:373-7. 